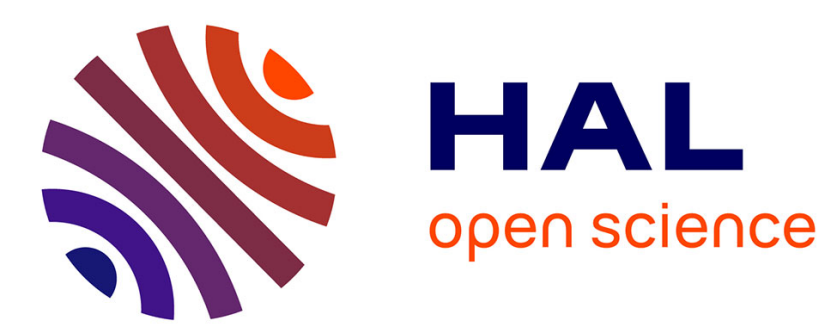

\title{
Les troubles moteurs chez les enfants dyslexiques: revue de travaux et perspectives
}

\author{
Marianne Jover, Stéphanie Ducrot, Andrea Huau, Stéphanie Bellocchi,
} Florence Brun-Henin, Josette Mancini

\section{- To cite this version:}

Marianne Jover, Stéphanie Ducrot, Andrea Huau, Stéphanie Bellocchi, Florence Brun-Henin, et al.. Les troubles moteurs chez les enfants dyslexiques: revue de travaux et perspectives. Enfance, 2014, 4, pp.323-347. hal-01485962

\section{HAL Id: hal-01485962 \\ https://hal.science/hal-01485962}

Submitted on 21 Jan 2022

HAL is a multi-disciplinary open access archive for the deposit and dissemination of scientific research documents, whether they are published or not. The documents may come from teaching and research institutions in France or abroad, or from public or private research centers.
L'archive ouverte pluridisciplinaire HAL, est destinée au dépôt et à la diffusion de documents scientifiques de niveau recherche, publiés ou non, émanant des établissements d'enseignement et de recherche français ou étrangers, des laboratoires publics ou privés. 
Jover, M., Ducrot, S., Huau, A., Bellocchi, S., Brun-Hénin, F., Mancini, J. (2013). Les troubles moteurs chez les enfants dyslexiques : revue de travaux et perspectives. Enfance, 4, 323-347. DOI: http://dx.doi.org/10.4074/S0013754513004023

\section{Les troubles moteurs chez les enfants dyslexiques : revue de travaux et perspectives Motor disorders in dyslexic children: a review and some perspectives}

Jover Marianne, Aix-Marseille Université, PsyCLE EA 3273, 13621, Aix en Provence, France Ducrot Stéphanie, Aix-Marseille Université, CNRS, LPL UMR 7309, 13100, Aix en Provence, France Huau Andréa, Aix-Marseille Université, PsyCLE EA 3273, 13621, Aix en Provence, France Bellocchi Stéphanie, Aix-Marseille Université, PsyCLE EA 3273, LPL UMR 7309, 13100, Aix en Provence, France

Brun-Hénin Florence, APHM, Hôpital de la Timone, CERTA, 13385, Marseille, France Mancini Josette, APHM, Hôpital de la Timone, CERTA, 13385, Marseille, France

Résumé : Introduction. La dyslexie est un trouble développemental très fréquemment associé à des troubles moteurs de différente nature. Cet article propose une revue des travaux portant sur la motricité des enfants et des adultes dyslexiques. Apports de la littérature. Dans un premier temps, nous constatons que la comorbidité entre dyslexie et trouble de l'acquisition des coordinations (TAC) est très fréquente. Par ailleurs, les recherches montrent que ces patients présentent des dysfonctionnements moteurs que l'on peut qualifier de subcliniques. Ces travaux peuvent être catégorisés en fonction des domaines de la motricité sur lesquels ils portent : contrôle de l'équilibre, motricité fine et motricité oculaire. Discussion. Nous développons à l'issue de cette revue deux théories explicatives de ces particularités, celle du développement cérébral atypique (Kaplan, Wilson, Dewey, \& Crawford, 1998) et celle du déficit d'apprentissage procédural (Nicolson \& Fawcett, 2007). Les travaux sur la comorbidité entre les troubles des apprentissages scolaires et non scolaires doivent, de notre point de vue être renforcés. Conclusion. Une approche plus intégrative pourrait permettre de mieux comprendre la cooccurrence entre les troubles neuro-développementaux.

Mots clés: dyslexie, trouble de l'acquisition de la coordination, coordination motrice, comorbidité

Summary: Introduction. Dyslexia is a developmental disorder frequently associated with motor disorders. This article offers a review of works on motor skills of children and adults with 
dyslexia. Literature findings. First, comorbidity between dyslexia and developmental coordination disorder (DCD) is very common. Moreover, studies show that these patients have motor dysfunction that can be described as subclinical. This paper categorizes the studies according to areas of motor skills: balance control, fine motor control and eye movements. Discussion. At the end of this review, we develop two theories advanced to explain motor disorders in dyslexia: the atypical brain development (Kaplan, et al., 1998) and the procedural learning disorder (Nicolson \& Fawcett, 2007). In our view, research focused on comorbidity between learning disabilities and motor disorders should be strengthened. Conclusion. A more integrative approach could help to better understand the cooccurrence between neurodevelopmental disorders.

Key words: dyslexia, developmental coordination disorder, motor coordination, comorbidity 


\section{Introduction}

La dyslexie est un trouble spécifique des apprentissages également décrit sous le terme de trouble spécifique de la lecture. Les critères diagnostiques sont 1) un niveau de lecture (exactitude, rapidité ou compréhension) significativement inférieur à celui escompté compte-tenu de l'âge, des capacités intellectuelles et de la qualité de l'enseignement dispensé, 2) une interférence de cette difficulté sur le parcours scolaire et l'adaptation, et 3) l'absence de déficit sensoriel pouvant expliquer les difficultés (American Psychiatric Association, 2003; Habib \& Joly-Pottuz, 2008; Organisation Mondiale de la Santé, 1994).

Le DSM5 a récemment intégré la dyslexie comme une des trois dimensions du Trouble spécifique des apprentissages, avec la dyscalculie et le trouble de l'expression écrite. Cette décision s'inscrit dans une approche dimensionnelle globale des troubles neuro-développementaux, fondée notamment sur la cooccurrence mais également sur le partage de mécanismes communs (Andrews, Pine, Hobbs, Anderson, \& Sunderland, 2009). Cet article propose de nourrir cette approche dimensionnelle en réalisant une synthèse des travaux sur la motricité des enfants et des adultes dyslexiques. Après avoir envisagé les cas de comorbidité entre dyslexie et trouble de l'acquisition de la coordination (TAC), nous développerons cet écrit autour des particularités motrices des patients dyslexiques dans les domaines de l'équilibre, des coordinations manuelles fines (graphomotricité) et de la motricité oculaire. Nous aborderons, pour terminer, les hypothèses permettant de les expliquer et les perspectives de recherche ouvertes par cette revue.

\section{De la cooccurrence entre dyslexie et trouble de l'acquisition de la}

\section{coordination}

Le trouble de l'acquisition des coordinations (TAC) est défini par le DSM5 comme une atteinte significative de la réalisation des activités quotidiennes nécessitant une coordination motrice. Le sujet présente des performances motrices inférieures à ce que l'on pourrait attendre compte-tenu de son âge chronologique et de ses capacités intellectuelles. Ce trouble neuro-développemental n'est pas dû à une affection somatique avérée et peut toucher différents aspects de la motricité, depuis la marche, les coordinations globales, l'équilibre, jusqu'aux coordinations motrices fines et l'écriture (American Psychiatric 
Association, 2003). Le TAC est également connu en France sous le terme de dyspraxie de développement (Polatajko, Fox, \& Missiuna, 1995).

La cooccurrence entre dyslexie et TAC est fréquente et rapportée dans de nombreuses publications (tableau 1). Lorsque I'on prend comme point de départ des échantillons d'enfants dyslexiques, le TAC est associé avec une fréquence de 9.7 à $70 \%$ selon les critères diagnostiques et l'origine des populations étudiées. Par ailleurs, lorsqu'on considère les recherches basées sur des populations d'enfants TAC, le taux de cooccurrence oscille entre 29 et $70 \%$. La grande variabilité de ces proportions est saisissante, particulièrement dans le cas où l'échantillon source est composé d'enfants dyslexiques. La présence de soustypes dans chacun de ces troubles neuro-développementaux explique probablement ces disparités (Bailey, Manis, Pedersen, \& Seidenberg, 2004; Peterson, Pennington, \& Olson, 2013).

\begin{tabular}{|c|c|c|}
\hline Publication & Echantillon source & Proportion de cooccurrence \\
\hline \multicolumn{3}{|c|}{ Troubles de la lecture } \\
\hline Critchley $(1974$, cité par Albaret, 2008) & 125 dyslexiques & $27 \%$ maladroits \\
\hline Mæland \& Søvik (1993) & 27 dyslexiques & $22 \% \mathrm{TAC}$ \\
\hline Kaplan et al. (1998) & 71 dyslexiques & $63 \% \mathrm{TAC}$ \\
\hline Kaplan, Dewey, Crawford, \& Wilson (2001) & 126 dyslexiques & $16 \%$ TAC \\
\hline Iversen, Berg, Ellertsen, \& Tonnessen (2005) & 20 dyslexiques & $60-70 \%$ TAC \\
\hline Iversen, et al. (2005) & 17 mauvais lecteurs & $53-58.9 \%$ TAC \\
\hline McPhillips \& Sheehy (2004) & 41 mauvais lecteurs & $20-34 \%$ TAC \\
\hline Kooistra, Crawford, Dewey, Cantell, \& Kaplan (2005) & 63 dyslexiques & $37 \%$ TAC \\
\hline Habib (INSERM, 2007) & 177 dyslexiques & $31 \%$ TAC \\
\hline Chaix et al. (2007) & 58 dyslexiques (phonologiques) & $40 \%-57 \%$ TAC \\
\hline Ho, Chan, Leung, Lee, \& Tsang (2005) & 31 dyslexiques & $9.7 \% \mathrm{TAC}$ \\
\hline \multicolumn{3}{|c|}{ Troubles de la motricité } \\
\hline Gubbay (1965, cité par Albaret, 2008) & 21 maladroits & $29 \%$ mauvais lecteurs \\
\hline Henderson \& Hall (1982) & 16 maladroits & $50 \%$ mauvais lecteurs \\
\hline Fletcher-Flinn, Elmes, \& Strugnell (1997) & $28 \mathrm{TAC}$ & $68 \%$ mauvais lecteurs \\
\hline Kaplan et al. (1998) & $81 \mathrm{TAC}$ & $56 \%$ dyslexiques \\
\hline O'Hare \& Khalid (2002) & $23 \mathrm{TAC}$ & $70 \%$ mauvais lecteurs \\
\hline Ho et al. (2005) & $21 \mathrm{TAC}$ & $42.8 \%$ dyslexiques \\
\hline Habib (INSERM, 2007) & 19 TAC & $63 \%$ dyslexiques \\
\hline
\end{tabular}

Tableau 1 : Synthèse des recherches explorant la cooccurrence de trouble de la lecture et de la motricité dans des échantillons composés soit d'enfant mauvais lecteurs/dyslexiques, soit d'enfant maladroits/TAC (selon la terminologie des auteurs). Les fourchettes de pourcentage rendent compte de la différence de critères utilisés par les auteurs pour identifier un TAC.

Au-delà de la stricte association morbide (i.e. du diagnostic des deux pathologies), le type de difficultés motrices rencontré par les enfants mauvais lecteurs ou dyslexiques a largement été exploré à l'aide de tests et d'échelles composites standardisés distinguant divers domaines moteurs (BOTMP, Bruininks, 1978; M-ABC, S. E. Henderson \& Sugden, 1992). Kooistra et al. (2005) ont ainsi montré que si les scores bruts au BOTMP étaient inférieurs aux contrôles chez les enfants dyslexiques, $48 \%$ des enfants dyslexiques présentent un score pathologique au niveau de la motricité globale contre $18 \%$ pour la motricité manuelle. De même, les travaux avec le M-ABC montrent que les enfants avec un bas niveau de lecture présentent des 
difficultés spécifiques aux items de dextérité manuelle et d'équilibre alors que le score dans les tâches de maîtrise de balle est identique à celui des bons lecteurs (enfants de 11-12 ans, Iversen, et al., 2005; enfants de 9-10 ans, McPhillips \& Sheehy, 2004). Avec une méthodologie d'analyse différente, Vuijk et al. (2011) ont cependant observé un lien significatif et positif (.18) entre le score à un test de lecture et celui aux items de maîtrise de balle du M-ABC chez des enfants de 7 à 12 ans présentant des troubles spécifiques des apprentissages scolaires. Avec une méthode d'analyse comparable, nous avons également relevé un lien positif et significatif entre la qualité de la lecture mesurée à l'aide du test de l'Alouette (Lefavrais, 2005) et la qualité globale de la motricité mesurée à l'aide du M-ABC (Soppelsa \& Albaret, 2004) dans un groupe d'enfants dyslexiques, TAC et tout-venant âgés de 8 à 12 ans. L'analyse indépendante des trois souséchelles du M-ABC ne permet toutefois pas de conclure à lien plus prononcé entre l'une d'entre elle et les difficultés de lecture dans cette recherche préliminaire (Jover, Huau, \& Velay, 2011).

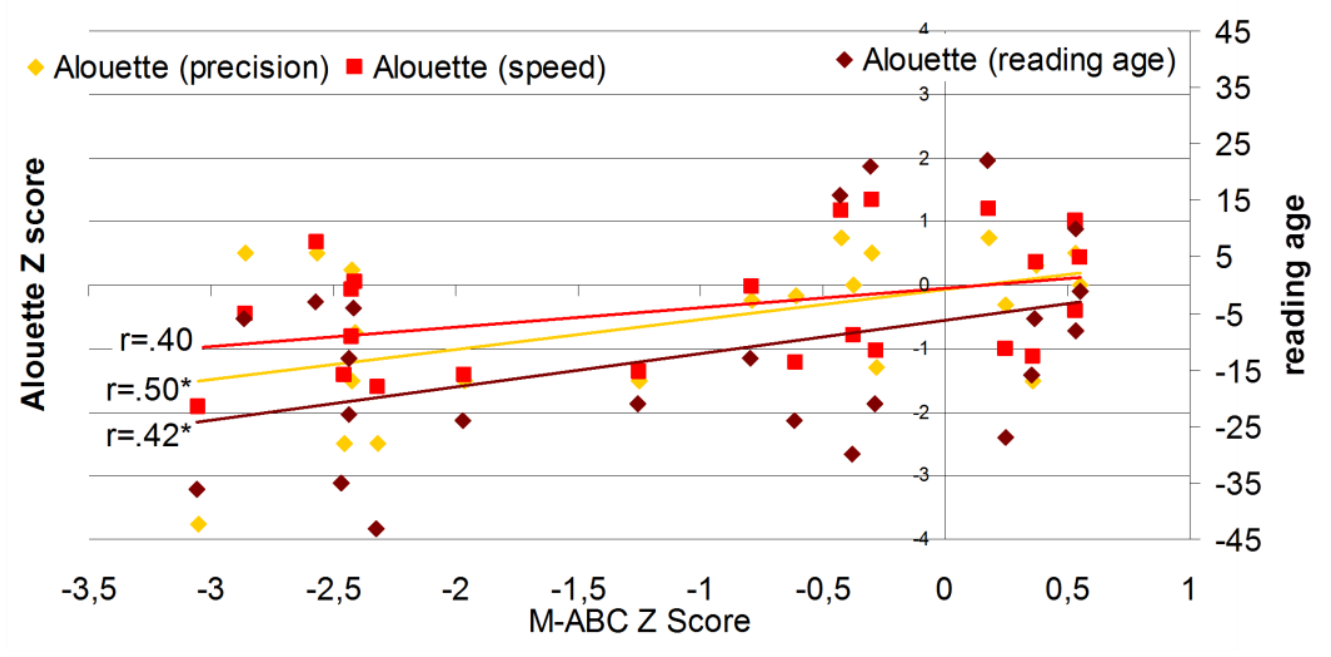

Figure 1 : Représentation graphique de la corrélation obtenue entre les scores $Z$ à l'Alouette et au $M-A B C$ dans un groupe d'enfants âgés de 8 à 12 ans et composé de 8 enfants dyslexiques, 8 enfants TAC et 8 enfants contrôles (d'après Jover, et al., 2011).

Ces aspects moteurs ne sont toutefois pas les seuls à avoir été explorés chez les enfants dyslexiques ou mauvais lecteurs. Ainsi, en utilisant le VMI (Beery Test of Visual-Motor Integration, Beery, 1989), qui est un test de copie de figures géométriques impliquant aussi les habiletés visuospatiales, Kooistra et al. (2005) ont observé que $44.4 \%$ des enfants dyslexiques de leur échantillon présentaient un score pathologique.

Les tests et échelles standardisés présentent le grand intérêt d'obtenir des données comparables dans différents échantillons par différents auteurs de pays variés. Leur utilisation dans ce contexte pose cependant plusieurs limites, liées à leurs objectifs. En effet, les tâches sélectionnées dans les tests le sont 
pour évaluer des performances des sujets et les comparer à une norme, et non pour comprendre les mécanismes responsables des déficits lorsqu'ils sont présents. De plus, la qualité des mesures est relativement limitée puisqu'ils produisent des scores, et très rarement des données fines ou des mesures continues. II est, dès lors, très utile de s'orienter également vers les travaux expérimentaux pour comprendre la nature des difficultés motrices rencontrées par les enfants dyslexiques.

\section{Etude expérimentale des difficultés motrices chez les patients}

\section{dyslexiques}

Dans la continuité des observations cliniques réalisées au travers d'épreuves standardisées, nombreuses sont les recherches qui ont exploré par des méthodes expérimentales les domaines de la motricité paraissant dysfonctionnels chez les dyslexiques : le contrôle de l'équilibre, la motricité fine et la motricité oculaire.

\subsection{L'hypothèse d'un trouble du contrôle de l'équilibre dans la dyslexie}

Plusieurs équipes ont approfondi l'analyse des performances des dyslexiques dans des tâches sollicitant le contrôle de l'équilibre, qui parait spécifiquement altéré chez ces patients (Haslum \& Miles, 2007).

Nicolson et Fawcett ont réalisé une série de travaux chez des enfants dyslexiques de 8, 12 et 16 ans et des enfants contrôles appariés en âge. Les tâches utilisées sont diverses : équilibre sur 1 pied ou sur 2 pieds, yeux fermés ou ouverts, avec ou sans double tâche cognitive. Les résultats montrent que les enfants dyslexiques se distinguent des non-dyslexiques dans les situations complexes uniquement (yeux fermés, double tâche). Dans la situation de double tâche, leurs performances sont tellement affectées que les enfants les plus âgés sont moins efficaces que les enfants contrôles les plus jeunes (Nicolson \& Fawcett, 1994). En analysant les tailles d'effet des différences entre enfants dyslexiques et contrôles, ces auteurs montrent que les tâches d'équilibre complexes discriminent le mieux les deux groupes, après l'âge d'orthographe (spelling age) et avant l'âge de lecture. Ainsi, 81 à $84 \%$ des enfants dyslexiques présentent des performances préoccupantes dans la tâche d'équilibre avec double tâche et la tâche d'équilibre les yeux fermés, contre 13 à 16\% chez les enfants contrôles (Nicolson \& Fawcett, 1995). Dans une procédure comparable, Fawcett, Nicolson, \& Dean (1996) montrent également que les enfants dyslexiques âgés de 10, 14 et 18 ans restent stables moins longtemps lorsqu'ils sont debout les yeux fermés et les pieds serrés, et qu'ils présentent des 
difficultés à récupérer leur équilibre après une légère poussée dans le dos. Ces résultats ont ensuite été globalement répliqués dans des protocoles légèrement différents, chez les enfants et les adultes par des auteurs de la même équipe (Fawcett \& Nicolson, 1999; Fawcett, Nicolson, \& Maclagan, 2001; Needle, Fawcett, \& Nicolson, 2006; Stoodley, Fawcett, Nicolson, \& Stein, 2005) ou par d'autres (Moe-Nilssen, Helbostad, Talcott, \& Toennessen, 2003; Pozzo et al., 2006; Vieira, Quercia, Michel, Pozzo, \& Bonnetblanc, 2009; Yap \& van der Leij, 1994), qui montrent tous une atteinte du contrôle de l'équilibre dans la dyslexie.

Ces difficultés d'équilibre ne sont toutefois pas unanimement retrouvées. Wimmer, et al. (1999), Raberger et Wimmer (2003), Ramus et al. (2003) ou Stoodley et al. (2006) ont échoué à montrer un déficit de l'équilibre dans une situation de double tâche chez des enfants ou des adultes dyslexiques lorsqu'ils contrôlaient la présence de troubles attentionnels. Pour ces auteurs, les difficultés d'équilibre constitueraient un signe de l'association de la dyslexie avec un trouble déficitaire de l'attention avec ou sans hyperactivité (TDAH) ou un TAC.

Face à ces résultats peu systématiques, Rochelle et Talcott (2006) ont réalisé une méta-analyse afin de quantifier l'importance des troubles de l'équilibre dans la dyslexie. Celle-ci confirme la forte incidence des troubles de l'équilibre dans ce trouble $(\mathrm{d}=.64)$ mais les tailles d'effets sont très variables entre les recherches. Pour les auteurs, la présence de comorbidités dans certaines recherches, ainsi que le contrôle limité des scores d'intelligence des sujets modèrent cet effet et contribuent probablement à brouiller le tableau.

\subsection{Motricité manuelle, écriture et dyslexie}

Les troubles de la motricité fine, et notamment graphomoteurs, peuvent apparaitre de façon relativement indépendante des troubles moteurs plus globaux et méritent d'être explorés en tant que tels (Jover, 2012).

Les coordinations manuelles fines ont été analysées dans des tâches extrêmement variées chez des enfants et des adultes dyslexiques: manipulation (Télécran, pegboard, perles), dessin ou écriture. Gladstone et al. (1989) et Moore et al. (1995) ont utilisé un Télécran, avec lequel les personnes doivent déplacer un curseur sur un écran à l'aide de molettes en contrôlant les déplacements verticaux et horizontaux. Les enfants et les adultes dyslexiques se distinguent des contrôles lorsque la tâche se complique, c'est-à-dire lorsque les deux mains doivent réaliser des mouvements en miroir. Les tâches de pegboard impliquent, quant à elles, de placer ou déplacer des chevilles dans une planche à trou. Si des différences entre enfants 
dyslexiques et enfants tout-venants apparaissent parfois avec ce dispositif, les recherches ne sont pas unanimes. Pour Leslie et al. (1985), la différence entre enfants dyslexiques et contrôles est significative dans les conditions unimanuelles uniquement et plus particulièrement avec la main gauche. Nicolson et Fawcett (1994) montrent toutefois que les difficultés des enfants dyslexiques disparaissent avec l'âge, ou lorsque les enfants sont comparés à des contrôles de même âge de lecture (Fawcett \& Nicolson, 1995). Wimmer et al. (1998) et Chaix et al. (2007) n'observent pas non plus de différence entre dyslexiques et contrôles dans cette tâche. Chez l'adulte, Stoodley et Stein (2006) notent toutefois que les dyslexiques sont plus lents, et ce d'autant plus que leur déficit en lecture est important. Enfin, dans des tâches d'enfilage de perles, Nicolson et Fawcett (Fawcett \& Nicolson, 1995; Nicolson \& Fawcett, 1994) ainsi que Ramus et al. (2003) ont relevé des performances significativement moins bonnes chez les enfants dyslexiques que chez les enfants contrôles, même s'ils sont appariés sur leur âge de lecture. II semble donc que si les sujets dyslexiques se trouvent parfois en difficultés dans les tâches manuelles, cet effet ne soit ni général, ni consensuel. Ici encore, la variabilité des profils cliniques et le niveau de difficulté des tâches expliquent probablement les résultats contradictoires de ces recherches.

La graphomotricité, du fait de son rapport avec l'écriture et donc avec la lecture, a été largement étudiée chez les enfants et les adultes dyslexiques. Même lorsque les tâches sont sans contenu langagier, plusieurs recherches montrent que les enfants dyslexiques se distinguent des enfants contrôles (Iversen, et al., 2005; McPhillips \& Sheehy, 2004). Par exemple, l'item de la fleur du M-ABC (S. E. Henderson \& Sugden, 1992) dans lequel les enfants doivent tracer un trait entre deux lignes est celui qui distingue le mieux les populations d'enfants dyslexiques et non dyslexiques et il est d'autant moins bien réussi que la dyslexie est sévère (Iversen, et al., 2005). En analysant et comparant les productions graphiques d'enfants dyslexiques, et contrôles, nous avons également relevé des paramètres graphomoteurs particuliers chez les enfants dyslexiques, comme la pression exercée sur le stylo, significativement supérieure au groupe contrôle lors d'une tâche de précision comparable à l'item de la fleur du M-ABC (Huau, Velay, \& Jover, 2012).

L'écriture repose sur des processus phonologiques, syntaxiques et sémantiques en amont de la formation d'une trace (van Galen, 1991). Les recherches portant spécifiquement sur le versant graphomoteur de l'écriture donnent des résultats contradictoires chez les dyslexiques. Tout d'abord, les évaluations standardisées de la qualité et de la vitesse d'écriture ont permis de montrer que la dyslexie est souvent associée à la dysgraphie. Chaix et al. (2007) identifient ainsi 21\% de dysgraphiques dans un groupe d'enfants 
présentant une dyslexie phonologique «pure », c'est-à-dire ne présentant ni TDAH, ni atteinte motrice générale. Habib (2003, cité par INSERM, 2007) repère quant à lui 14\% d'enfants dysgraphiques dans une population de 177 enfants dyslexiques. A l'aide d'une batterie d'évaluation globale évaluant l'écriture, l'orthographe et la composition écrite, Berninger, Nielsen, Abbott, Wijsman, et Raskind (2008) ont noté que la dimension graphomotrice de l'écriture était altérée chez les dyslexiques. C'est également ce qu'a observé Brun-Hénin (2007) chez des enfants et des adultes dyslexiques à l'aide de la tâche de Gobineau (de Ajuriaguerra et al., 1964). Elle relève, en outre, chez les adultes une corrélation entre les scores de lecture et les scores d'écriture, traduisant une certaine proportionnalité entre les troubles.

L'analyse des traces écrites à l'aide d'une tablette graphique et d'un système informatique permet une exploration plus fine de la graphomotricité. Avec ce type de dispositif, Lam, Au, Leung et Li-Tsang (2011) notent que les enfants dyslexiques écrivent plus lentement, moins précisément, qu'ils font des lettres plus grandes et plus variables en taille que les enfants contrôles. Brun-Hénin (2007) repère également une lenteur plus marquée chez les enfants dyslexiques lors de l'écriture d'un texte. Borella et al. (2011) observent des différences moins marquées, dans une tâche plus simple consistant à reproduire une séquence de lettres alternées (l et e): les enfants dyslexiques et contrôles se distinguent uniquement si l'on considère la variabilité intra-individuelle de la vitesse. Plusieurs recherches échouent toutefois à montrer un effet de la dyslexie sur la dimension graphique de l'écriture. Velay et al. (2009) et Paz-Villagrán, Gilhodes, et Velay (2013) n'ont pas détecté de différence lors de la production de lettres isolées, groupées, de mots ou lors de l'apprentissage d'un nouveau caractère entre enfants dyslexiques non dysgraphiques et enfants contrôles. Chez des adultes dyslexiques, Brun-Hénin (2007) n'observe également pas de modification de la cinématique de l'écriture. Dans une recherche récente, ces mêmes auteurs ont cependant montré que si les enfants dyslexiques ne sont pas plus lents que les enfants contrôles lorsqu'ils écrivent une phrase en lettres cursives ou majuscules, ils présentent, en revanche, des intervalles temporels significativement supérieurs, entre les mots et entre les lettres (Brun-Hénin, Velay, Beecham, \& Cariou, 2013). Ces résultats concordent avec ceux obtenus par Huau et al. (2012) dans une tâche d'écriture de mots présentée à des enfants TAC, dyslexiques et tout-venants. Les analyses de régression réalisées à partir des scores aux tests d'écriture (BHK, Charles, Soppelsa, \& Albaret, 2003), de lecture (Alouette, Lefavrais, 2005) et au M-ABC (Soppelsa \& Albaret, 2004) montrent que plus les enfants ont des difficultés en lecture, plus ils réalisent des pauses le stylo en l'air et plus celles-ci sont longues. 
Les résultats disponibles chez les enfants ou les adultes dyslexiques sont donc contradictoires mais suggèrent plutôt, en tous cas au niveau clinique, que les troubles graphomoteurs et de l'écriture sont présents, même si peu intenses, chez ces patients. L'analyse de la cinématique des tracés fait penser que certaines caractéristiques de l'écriture permettent de différencier les sujets dyslexiques des sujets toutvenants, mais dans des tâches un peu complexes et en utilisant des indices fins des procédures graphomotrices.

\subsection{Attention visuelle, oculomotricité et dyslexie}

L'activité de lecture fait intervenir trois types de mouvements oculaires: les saccades, immédiatement suivies par des périodes de fixation où l'œil reste immobile, la vergence et les mouvements combinés associant les saccades et les mouvements de vergence.

\section{Troubles neurovisuels et visuo-moteurs de bas niveau}

La recherche de caractéristiques visuelles ou visuo-motrices particulières chez le dyslexique a pris un nouvel essor après la découverte d'anomalies du système magnocellulaire (i.e. système permettant le traitement des basses fréquences spatiales - contours des mots par exemple - impliqué aussi dans la gestion des mouvements oculaires) au sein des voies visuelles lors d'autopsie de cerveau de dyslexiques (Stein \& Walsh, 1997). Les dyslexiques souffriraient ainsi de perturbations de la vision des contrastes et de la sensibilité au mouvement (Cornelissen, Richardson, Mason, Fowler, \& Stein, 1995; Lovegrove, Martin, \& Slaghuis, 1986), d'une instabilité de la fixation oculaire engendrant des distorsions, des déplacements et des superpositions de lettres et de mots (Chase \& Stein, 2003; Stein, 2001), ainsi que des troubles de la poursuite oculaire, essentiellement de gauche à droite (Eden, Stein, Wood, \& Wood, 1994). Stein et al. (1988) ont, par ailleurs, montré des difficultés de convergence chez les dyslexiques et des anomalies de divergence tant en vision de près qu'en vision de loin, dues vraisemblablement à un défaut de coordination des saccades oculaires des deux yeux (voir aussi Kapoula et al., 2007). L'existence d'un déficit de la coordination binoculaire, non spécifique de la lecture a également été mis en évidence chez les dyslexiques (Bucci, Bremond-Gignac, \& Kapoula, 2008; Kirkby, Blythe, Drieghe, \& Liversedge, 2011).

L'existence de ces troubles de bas niveau et leur lien avec la dyslexie font l'objet de nombreuses controverses dans la littérature, aussi bien théoriques que méthodologiques. II semble d'une part que la proportion de dyslexiques présentant des troubles visuels de ce type soit faible (de 0 à $25 \%$; Ramus et al., 2003). De plus, la nature de la relation entre ces troubles et les difficultés d'apprentissage de la lecture est le 
plus souvent considérée comme non directe et peu de théories aujourd'hui sont aptes à expliquer clairement en quoi un déficit de ces traitements pourrait gêner l'apprentissage de la lecture et quel profil de performance en lecture devrait être attendu dans ce cas. Enfin, aucune étude n'a montré que les traitements de bas niveau en question se développaient plus particulièrement lors de la confrontation avec le langage écrit, ni que ces traitements étaient d'autant plus efficaces que l'expertise en lecture était élevée ou encore que des programmes d'entraînement spécifiques parviendraient à améliorer la lecture des sujets dyslexiques (Kavale \& Mattson, 1983).

\section{Anomalies des mouvements oculaires et déficits visuo-attentionnels spécifiques à l'activité de lecture}

La mobilité du regard n'est toutefois pas le seul moteur de la vision fine. Les mouvements des yeux et l'attention visuelle sont aussi impliqués. Ainsi, des capacités visuo-attentionnelles fines ${ }^{1}$ sont également importantes car un certain nombre de contraintes anatomo-fonctionnelles du système visuel limitent la prise d'information (voir pour une revue Ducrot \& Lété, 2008). On admet généralement que les lecteurs occidentaux traitent le texte à partir d'une fenêtre attentionnelle relativement petite, limitée à la ligne de texte en train d'être lue, et qui s'étend approximativement du début du mot fixé à 14-15 caractères sur la droite de la lettre fixée. A l'intérieur de cette fenêtre, deux types de traitement sont effectués : (1) un traitement fovéal de haute acuité, qui permet l'identification du mot en train d'être fixé et (2) un traitement parafovéal qui renseigne principalement sur la forme et la longueur des mots suivants, ce qui permet ainsi de guider l'œil et de déterminer le lieu de la prochaine fixation. Lors de l'apprentissage de la lecture, l'enfant va ainsi devoir apprendre à se positionner correctement sur les mots, extraire l'information qui est en train d'être fixée, programmer une saccade pour se positionner sur le mot suivant (voir pour une présentation détaillée Ducrot, Pynte, Ghio, \& Lete, 2013).

De nombreuses études ont montré que les mouvements oculaires de sujets dyslexiques dans des tâches de lecture de mots isolés, de pseudo-mots ou même de phrases sont différents par rapport à ceux d'une population de même âge : les fixations sont plus longues et plus nombreuses, les saccades plus courtes, la proportion de régressions est supérieure, et les mouvements de retour à la ligne sont souvent erratiques (pour une revue Bellocchi, Muneaux, Bastien-Toniazzo, \& Ducrot, 2013; Biscaldi, Gezeck, \& Stuhr, 1998; Hawelka, Gagl, \& Wimmer, 2010; Hutzler \& Wimmer, 2004; Rayner, 1986). Les dyslexiques présentent également un empan perceptif un peu plus petit que les normo-lecteurs (Rayner, Pollatsek, \& Bilsky, 1995).

\footnotetext{
${ }^{1}$ Les aptitudes d'attention visuelle sélective se développent vers cinq-six ans et permettent au sujet de guider ses yeux vers un point précis, tout en filtrant les informations non pertinentes.
} 
Comme Rayner (1998) l'expliquait, il n'est pas possible à ce jour d'affirmer que le patron atypique des mouvements oculaires le plus souvent observé chez les dyslexiques soit la cause plutôt que la conséquence de leurs difficultés de lecture. Ainsi, on retrouve une configuration de mouvements identiques (saccades plus courtes, fixations plus longues, régressions) chez des enfants plus jeunes de même niveau de lecture (Haikio, Bertram, Hyona, \& Niemi, 2009; Rayner, 1986) ou encore lorsqu'on présente au lecteur expert un texte difficile à lire (J. M. Henderson \& Ferreira, 1990; Rayner, Slattery, \& Belanger, 2010). D'autre part, les particularités observées en situation de lecture disparaissent lorsque la tâche n'implique pas de lire. Ainsi, Hutzler, et al. (2006) montrent un pattern oculomoteur très atypique en situation de lecture de séquences de pseudo-mots, pattern qui se normalise lorsqu'on demande simplement aux dyslexiques de repérer les séquences de deux lettres identiques dans une situation de non-lecture en tout point similaire (voir également De Luca, Di Pace, Judica, Spinell, \& Zoccolotti, 1999; Hutzler \& Wimmer, 2004; Prado, Dubois, \& Valdois, 2007).

Néanmoins, certains auteurs ont pu montrer des problèmes spécifiques en ce qui concerne le guidage oculaire. On sait en effet que pour qu'un mot soit traité de la meilleure façon possible, il faut que l'œil se pose dans une zone à mi-chemin entre le début et le milieu du mot. II semble que cette position soit choisie de façon stratégique afin d'optimiser le traitement (O'Regan, Levy-Schoen, Pynte, \& Brugaillere, 1984; Rayner, 1979). Or, chez les sujets dyslexiques, on constate que le point fixé n'est pas toujours idéalement choisi et les erreurs de positionnement sont plus fréquentes et occasionnent aussi, à la différence des enfants normo-lecteurs, une augmentation des refixations (Ducrot, et al., 2013; Hawelka, et al., 2010). Allant dans ce sens, une distribution anormale de l'attention de part et d'autre du point de fixation est rapportée, avec une sensibilité accrue à la distraction à droite du point de fixation et une réduction de l'attention à gauche du point de fixation (Facoetti et al., 2003; Facoetti \& Molteni, 2001). Les enfants dyslexiques ne parviendraient pas à contrôler la distribution de l'attention par une stratégie habituellement mise en place par les bons lecteurs pour restreindre le faisceau attentionnel en vision périphérique, entraînant un traitement atypique de l'information visuelle hors de la zone fovéale (Ducrot, Lété, Sprenger-Charolles, Pynte, \& Billard, 2003; Geiger, Lettvin, \& Zegarra-Moran, 1992). Ce phénomène de supériorité du traitement parafovéal chez les dyslexiques est une des hypothèses qui peut expliquer la grande sensibilité de cette population clinique au phénomène " d'encombrement perceptif », c'est-à-dire au masquage visuel de chaque lettre par celles qui l'entourent (e.g. Bellocchi, sous presse; Martelli, Di Filippo, Spinelli, \& Zoccolotti, 2009; Zorzi et al., 2012). D'autres travaux suggèrent que les particularités oculomotrices observées chez les dyslexiques seraient à 
mettre en lien avec un trouble spécifique de l'empan visuo-attentionnel, associé à une répartition atypique de l'attention visuelle (Bosse, Tainturier, \& Valdois, 2007; Valdois, Lassus-Sangosse, \& Lobier, 2012).

Dans ce contexte, il est important de noter que des progrès significatifs en lecture ont été observés après des remédiations ciblées sur le mécanisme oculomoteur et/ou attentionnel déficient. Par exemple, une rééducation centrée sur l'entraînement des capacités de traitement visuo-attentionnel s'est avérée très efficace chez les enfants dyslexiques (Launay \& Valdois, 2004; Lobier \& Valdois, 2010). Plus récemment, il a été montré que l'augmentation de l'espacement des lettres d'un mot et des mots d'un texte améliore la vitesse et la qualité de la lecture chez les enfants dyslexiques, et ce sans aucun entraînement préalable. Ils lisent alors en moyenne $20 \%$ plus vite et font deux fois moins d'erreurs (Zorzi, et al., 2012). Cette amélioration de la lecture pourrait être due au fait que cette technique permet d'une part de réduire " l'encombrement perceptif » et d'autre part d'augmenter la saillance des espaces entre les caractères dont on a montré qu'ils jouent un rôle particulièrement important dans la programmation saccadique et la position d'atterrissage de l'œil dans les mots (Ducrot et al., en préparation; Ducrot \& Pynte, 2002). Allant dans ce sens, Lehtimäki et Reilly (2005) ont montré qu'il était possible d'entraîner les jeunes enfants à contrôler leurs mouvements oculaires de façon à leur apprendre à atterrir à la position optimale pour traiter les mots.

\subsection{Synthèse}

Les troubles moteurs sont fréquents dans la dyslexie, ce qu'atteste la revue de littérature présentée, qui n'est, en outre, pas exhaustive (e.g. Poblano et al., 2002; Velay, Daffaure, Giraud, \& Habib, 2002; Viholainen et al., 2006). Le tableau qui se dégage est celui de personnes dyslexiques pouvant présenter des particularités motrices, mais à un niveau souvent subclinique, et face à des tâches plutôt complexes. Pour certains patients toutefois, les difficultés sont plus importantes, ou moins bien compensées, et nécessitent un diagnostic complémentaire de TAC ou de dysgraphie, ou une prise en charge en orthoptie.

Le caractère contradictoire de certains travaux ne doit pas oblitérer l'intérêt de la question posée par la présence de ces dysfonctionnements. En effet, l'explication théorique des dysfonctionnements présents dans la dyslexie est très débattue (Bishop \& Snowling, 2004; Nicolson, Fawcett, Brookes, \& Needle, 2010; Pennington, 2006; Ramus, Rosen, et al., 2003). Parmi les thèmes de discussion, le caractère unique ou multiple des déficits à l'origine de la pathologie, et en filigrane, la spécificité des dysfonctionnements cérébraux ou cognitifs, présents dans la dyslexie constituent un point fondamental. II est ainsi probable que la présence de troubles moteurs constitue un indice de la spécificité de certains sous-types de dyslexie, ou plus 
généralement, du multi-déterminisme de ce trouble neurodéveloppemental (Pennington, 2006; Peterson, et al., 2013). Les travaux autours de la comorbidité alimentent naturellement ces débats (trouble du langage oral et dyslexie, TDAH et dyslexie, TAC et dyslexie) en analysant la spécificité des mécanismes déficitaires dans la dyslexie par rapport à d'autres troubles neuro-développementaux.

\section{Cadres interprétatifs de la présence des troubles moteurs dans la dyslexie}

La fréquence et la nature des troubles moteurs identifiés dans la dyslexie et décrits dans cette revue de travaux renforcent l'hypothèse d'une communauté de déterminants entre la dyslexie et le TAC. Deux modèles théoriques évoquent cette hypothèse, dont l'un provient plutôt de la recherche sur le TAC (modèle du Développement Cérébral Atypique, Gilger \& Kaplan, 2001) et l'autre de recherches sur la dyslexie (modèle du déficit de l'apprentissage procédural, Nicolson, et al., 2010).

\subsection{Le modèle du Développement Cérébral Atypique}

Le modèle du Développement Cérébral Atypique (DCA, atypical brain development, Gilger \& Kaplan, 2001; Kaplan, et al., 2001; Kaplan, et al., 1998) a pour vocation de proposer une explication à la très fréquente cooccurrence des troubles spécifiques du développement en postulant une étiologie commune. Le DCA n'est pas un syndrome unitaire tel qu'avait été conçu le Dysfonctionnement Cérébral a Minima (Clements \& Peters, 1962), il s'agit au contraire de proposer une origine commune à des troubles développementaux différents dans leur expression. Le cerveau est conçu comme la base des apprentissages et des comportements et son développement, atypique, conduirait à des dysfonctionnements, ou encore à des fonctionnements particulièrement efficients ou hétérogènes (précocité intellectuelle, hyperlexie, TDAH...). Le caractère développemental du modèle est fondamental, avec une origine probablement prénatale ou précoce, et une expression symptomatique variable et éventuellement transitoire au cours du développement. (Gilger \& Kaplan, 2001; Gilger \& Wilkins, 2008). Le DCA inclue également l'idée d'un continuum de sévérité entre les troubles développementaux: la communauté des mécanismes responsables des troubles des apprentissages implique que dans sa forme sévère, un trouble des apprentissages est plus fréquemment associé à un autre trouble (Kaplan, Crawford, Cantell, Kooistra, \& Dewey, 2006). 
Les arguments en faveur de ce modèle relèvent essentiellement des travaux sur le taux de cooccurrence entre dyslexie, dysphasie, TAC et TDAH (tableau 1). Ce modèle permet également d'expliquer les nombreux sous-types observés dans une même entité nosographique, comme le TAC, ainsi que la grande variété des étiologies envisagées (Gilger \& Wilkins, 2008; Visser, 2003). II ne semble donc pas y avoir une région ou un système spécifiquement atteint dans une pathologie mais plutôt un fonctionnement atypique dont la nature varie sensiblement d'un tableau clinique à l'autre, avec cependant, de nombreux recouvrements. Si la contribution de ce modèle est fondamentale sur les principes qu'il contient, il présente toutefois le défaut d'être très flou sur les mécanismes impliqués dans chacun de ces troubles et leurs trajectoires développementales.

\subsection{Le modèle du déficit de l'apprentissage procédural}

Les nombreux travaux de Nicolson et Fawcett constituent, par leur cohérence, une piste sérieuse de compréhension de la dyslexie et des troubles moteurs associés à travers des hypothèses formulées sur deux niveaux d'explication, le premier cognitif, et le second cérébral (pour une revue détaillée Brun-Hénin, et al., 2013; Nicolson \& Fawcett, 2005).

\section{Modélisation cognitive de la dyslexie : le déficit d'automatisation}

Ces auteurs ont développé, dans les années 90, l'hypothèse qu'un déficit d'automatisation serait à l'origine de la dyslexie. L'automatisation est définie comme le processus par lequel, après une expérience pratique importante, des habiletés deviennent si fluides qu'elles ne nécessitent plus de contrôle conscient (Nicolson \& Fawcett, 1990). Pour ces auteurs, les problèmes de lecture et les difficultés phonologiques des enfants dyslexiques traduisent un niveau d'automatisation incomplet de l'apprentissage de la lecture. La difficulté d'automatisation des habiletés est alors envisagée comme un niveau de description cognitif des troubles observés chez les enfants dyslexiques, qu'ils soient langagiers ou moteurs. Dans cette perspective, et sachant que le manque d'automatisation peut être compensé par des stratégies diverses, les auteurs ont proposé à des enfants et des adultes dyslexiques des tâches de niveau de complexité croissant, et surtout ont eu recours à des protocoles de double tâche dans lesquels les ressources attentionnelles, plus sollicitées lorsque les habiletés ne sont pas automatisées, sont requises par une tâche complémentaire (compter à rebours,...). Comme nous l'avons développé dans la partie sur les troubles de l'équilibre, leurs résultats, et ceux d'autres auteurs également, montrent que les dyslexiques sont en difficulté dans la plupart des tâches 
motrices lorsqu'une deuxième tâche est présentée (Fawcett \& Nicolson, 1992; Fawcett, et al., 1996; Needle, et al., 2006; Nicolson \& Fawcett, 1990; Yap \& van der Leij, 1994).

\section{L'apport des travaux sur le cervelet}

Au niveau cérébral, l'hypothèse du déficit d'automatisation a été mise en lien avec celle d'un dysfonctionnement cérébelleux (Nicolson \& Fawcett, 1999, 2005; Nicolson, Fawcett, \& Dean, 2001). L’atteinte du cervelet ou des boucles cortico-cérébelleuses serait à l'origine des troubles phonologiques et des troubles moteurs observés dans la dyslexie. La localisation et l'étendue de l'atteinte détermineraient le profil des enfants : atteinte limitée au langage ou atteinte large s'étendant à la motricité (Nicolson \& Fawcett, 2006).

Cette hypothèse a été testée par la présentation de tâches impliquant le cervelet à des patients dyslexiques : mouvements oculaires et clignement de paupière, mouvements rapides, stabilité posturale et équilibre, apprentissage moteur implicite ; ainsi que par un certain nombre de recherches en imagerie cérébrale. Les résultats montrent qu'un certain nombre de patients dyslexiques présentent des performances déficitaires dans ces tâches. Par ailleurs, des spécificités anatomiques et des dysfonctionnements de l'activité cérébelleuse dans la dyslexie ont également été identifiés (pour une revue, Stoodley \& Stein, 2011, 2013).

De nombreuses critiques ont été formulées à propos de ce modèle (voir les débats dans Trends in Neurosciences, $24: 92001$ et dans Developmental Science, $9: 3$ 2006). Les dysfonctionnements repérés dans le cerveau des patients dyslexiques sont beaucoup plus étendus que le cervelet (Habib, 2000), qui pourrait alors seulement être conçu comme une structure recevant des informations dysfonctionnelles de régions corticales («le spectateur innocent», (Zeffiro \& Eden, 2001)). Enfin, les déficits cérébelleux ne peuvent pas être considérés comme un aspect central de la dyslexie : certains dyslexiques ne présentent aucun trouble moteur, tous les enfants présentant un trouble cérébelleux ne sont pas dyslexiques et les symptômes moteurs sont peu corrélés aux symptômes relatifs à l'alphabet (Nicolson \& Fawcett, 2007). Au final, l'hypothèse d'une contribution d'un dysfonctionnement cérébelleux à la dyslexie est forte. Cependant, l'implication du cervelet ne permet pas de statuer sur l'implication d'autres structures en amont (notamment corticales) et ni sur le lien de causalité entre dysfonctionnement cérébelleux et dyslexie (Stoodley \& Stein, 2011, 2013).

\section{Le modèle du déficit d'apprentissage procédural : prise en compte de la cooccurrence}

Les hypothèses du déficit d'automatisation et de l'implication cérébelleuse dans la dyslexie ont été réunies dans un modèle intégratif des troubles neuro-développementaux: le modèle du déficit 
d'apprentissage (Nicolson \& Fawcett, 2007, 2011). Les travaux expérimentaux et échanges théoriques autour de leurs propositions ont conduit ces auteurs à repenser leur modèle en termes d'apprentissage, au niveau cognitif, et de réseaux de neurones, au niveau cérébral (figure 2), modèle dans lequel les troubles neurodéveloppementaux seraient réunis sous la responsabilité d'un déficit du système d'apprentissage. Un dysfonctionnement des réseaux de neurones autour du cervelet, du cortex moteur ou des ganglions de la base conduirait à une réduction de la capacité à acquérir les compétences associées à ces régions. II en résulterait soit un retard global de développement, soit un trouble spécifique du développement, en fonction des circuits neuronaux impliqués et de l'importance de l'atteinte. Les atteintes du système d'apprentissage procédural perturberaient l'acquisition des habiletés motrices, induisant des troubles développementaux, soit dans les activités motrices séquentielles dans le cas d'atteintes du circuit cortico-striatal (TAC ou trouble du langage oral), soit dans les activités motrices nécessitant une synchronisation adaptative dans le cas d'atteintes du circuit cortico-cérébelleux (dyslexie, dysgraphie) (Nicolson \& Fawcett, 2011).

Les troubles neuro-développementaux sont donc à considérer à chaque fois à partir d'un symptôme central, mais également en prenant en compte les symptômes secondaires afin d'identifier les mécanismes dysfonctionnels et leurs étendues. Par exemple, la cooccurrence entre des problèmes moteurs et phonologiques indiquerait que les composantes motrices et langagières du système d'apprentissage procédural cortico-cérébelleux sont atteintes alors que l'absence de signe moteur suggèrerait que seules les composantes relatives au langage sont atteintes.

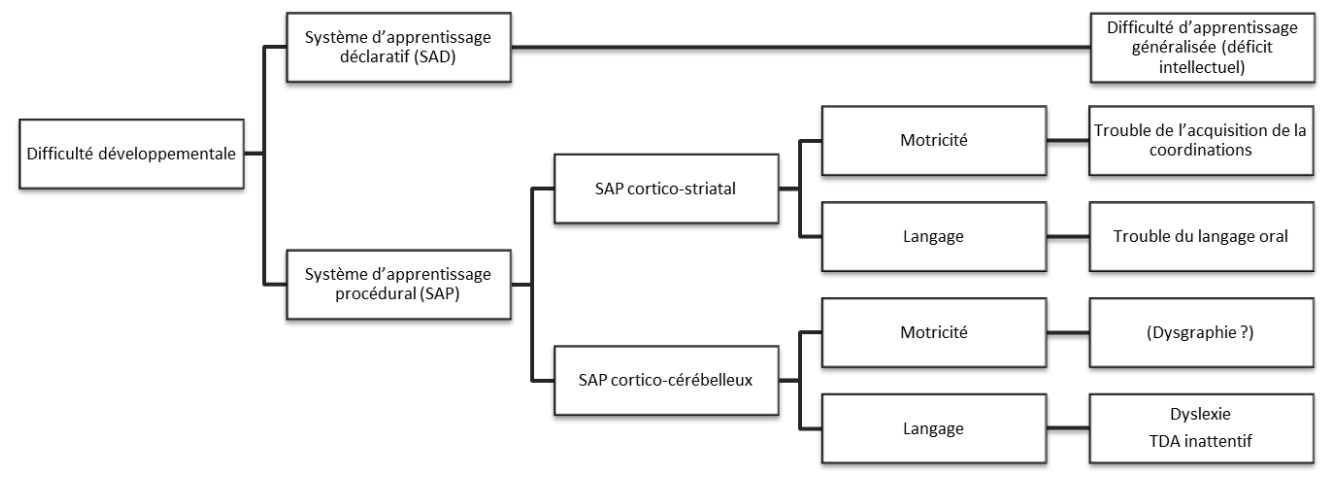

Figure 2 : Représentation schématique du modèle du déficit d'apprentissage procédural, faisant un lien entre les systèmes d'apprentissage, les réseaux neuronaux et les troubles neuro-développementaux ( $d$ 'après Nicolson et Fawcett, 2011).

Ce modèle intègre donc la comorbidité intrigante entre les désordres développementaux qui fait qu'avec les méthodes diagnostiques actuelles, les symptômes principaux d'une pathologie sont souvent les 
symptômes secondaires d'une autre, et vice-versa² (page 122, Nicolson \& Fawcett, 2011). En effet, les profils cliniques ne sont pas à considérer comme relevant d'une branche ou d'une autre mais il s'agit plutôt d'évaluer, dans le schéma très simplifié de la figure 2, l'importance de l'atteinte des différentes branches. Le caractère développemental de ces troubles rend aisément compte de cette dispersion des dysfonctionnements sur plusieurs niveaux (Nicolson \& Fawcett, 2011). Dans ce dernier article en date, Nicolson et Fawcett (2011) invoquent Newell (1973) et s'associent à l'hypothèse selon laquelle les troubles des apprentissages pourraient constituer des points sur un continuum (Kaplan, et al., 1998; Pennington, 2006).

De nombreuses recherches ont été récemment réalisées pour tester l'hypothèse du déficit d'apprentissage procédural dans la dyslexie. La majorité des recherches suggère que les sujets dyslexiques présentent un apprentissage défectueux mais les méthodologies sont variées et le tableau global encore confus (e.g. Bennett, Romano, Howard, \& Howard, 2008; Folia et al., 2008; Gabay, Schiff, \& Vakil, 2012a, 2012b; Menghini et al., 2008; Stoodley, Harrison, \& Stein, 2006; Vicari et al., 2005; Vicari, Marotta, Menghini, Molinari, \& Petrosini, 2003). Nous avons développé nos travaux en direction de cette hypothèse du déficit de l'apprentissage procédural en proposant à des enfants dyslexiques âgés de 8 à 12 ans des tâches d'apprentissage graphomoteur consistant en la reproduction d'une lettre inconnue provenant du sanscrit (Velay, et al., 2009). Pour tenir compte du phénomène de comorbidité et du gradient de sévérité, les enfants dyslexiques ou tout-venants, sont caractérisés par leurs scores à des échelles standardisées d'écriture (BHK, Charles, et al., 2003) et de lecture (Alouette, Lefavrais, 2005). Les analyses statistiques, de type GEE (Generalized Estimated Equation), permettent de montrer la contribution des capacités de lecture et d'écriture sur les paramètres mesurés durant la tâche à l'aide d'une tablette graphique. Nos résultats suggèrent que le niveau de lecture contribue significativement à expliquer l'évolution de certains paramètres au cours de l'apprentissage (nombre de pauses, Huau, Jover, \& Velay, en préparation).

\section{Perspectives}

La présence de troubles moteurs dans la dyslexie est un phénomène relativement bien connu dans la communauté des psychomotriciens et des orthophonistes francophones et la mention de "comorbidité » est

\footnotetext{
${ }^{2}$ The intriguing comorbidity between different developmental disorders that suggested that with current diagnostic methods primary symptoms of one disability were often secondary symptoms of another, and vice versa.
} 
la règle plus que l'exception (Albaret, 2008; Basse, Albaret, \& Chaix, 1999; Brun-Hénin, 2007). De nombreux travaux de recherche confirment les observations cliniques en analysant spécifiquement des tâches ou des fonctions motrices. Le contrôle de l'équilibre, les coordinations manuelles, l'écriture et la motricité oculaire sont ainsi fréquemment déficitaires chez les patients dyslexiques. II est dès lors important de comprendre la raison pour laquelle certains patients dyslexiques présentent des difficultés motrices significatives et d'autres non.

Parmi les cadres interprétatifs, le modèle du déficit d'apprentissage procédural permet de rendre compte du phénomène de comorbidité d'une façon probante, reposant à la fois sur des éléments comportementaux et cérébraux (Nicolson \& Fawcett, 2007, 2011). II s'inscrit également dans la dynamique actuelle de « réunification » des troubles neuro-développementaux (Andrews, et al., 2009; Kaplan, et al., 1998). Les troubles de l'équilibre et de la coordination prennent dans cette configuration, une grande importance : leur intensité et leur nature pourraient constituer des indicateurs clés du ou des systèmes responsables du trouble (Pennington, 2006; Pennington et al., 2012). De même, l'analyse de ces troubles en tenant compte des sous-types de dyslexie peut être extrêmement éclairante (Nicolson, et al., 2001; Peterson, et al., 2013; Stoodley \& Stein, 2011; Visser, 2003). Ainsi, le rapport INSERM publié en 2007 précisait déjà : Une des questions majeures encore non résolue est de savoir s'il existe une forme particulière de dyslexie qui accompagnerait de façon spécifique les troubles de la coordination sensori-motrice chez le dyspraxique ( $p$. 356, INSERM, 2007).

Depuis quelques années, des équipes de recherche se sont attachées à analyser conjointement les patients dyslexiques et TAC pour explorer les aspects qui seraient communs et les aspects qui seraient spécifiques à ces pathologies (Biotteau, Albaret, Lelong, \& Chaix, 2013; Brookes, Nicolson, \& Fawcett, 2007; Brun-Hénin, et al., 2013; Chaix, Biotteau, Vayssiere, Lelong, \& Albaret, 2013; Geuze \& Kalverboer, 1994; Jover, et al., 2011; O'Hare \& Khalid, 2002). Les résultats montrent, tout d'abord, que si enfants TAC et dyslexiques se ressemblent sur certains points, ces ressemblances sont limitées et surtout, les profils des enfants sont extrêmement variables au cœur de chaque pathologie. D'autre part, l'analyse de la comorbidité constitue actuellement un véritable défi. En effet, les enfants présentant des profils de comorbidité paraissent parfois présenter des performances moins bonnes que les troubles isolés, cumulant pour ainsi dire les difficultés des uns et des autres selon un gradient de sévérité (Brun-Hénin, et al., 2013; Jover, et al., 2011), et d'autres fois au contraire, des profils intermédiaires suggérant une trajectoire développementale spécifique 
dans le cas de la comorbidité (Biotteau, et al., 2013; Chaix, et al., 2013). Des recherches complémentaires et surtout des méthodologies adaptées doivent encore être développées pour appréhender cette question. II s'agit en effet, au niveau de la recherche comme au niveau des prises en charge, d'adopter une approche plus intégrative des pathologies neuro-développementales pour repenser l'actuelle dissociation qui persiste entre ces troubles et explorer la pertinence de l'approche dimensionnelle, telle la propose par exemple le DSM5 (Andrews, et al., 2009).

Conflit d'intérêt : aucun 


\section{Bibliographie}

Albaret, J.-M. (2008). La question de la comorbidité entre trouble de l'acquisition de la coordination (TAC) et trouble de la lecture. Fréquences, 19(4), 28-30.

American Psychiatric Association, A. P. A. (2003). DSM-IV, Manuel diagnostique et statistique des troubles mentaux. Paris: Masson.

Andrews, G., Pine, D. S., Hobbs, M. J., Anderson, T. M., \& Sunderland, M. (2009). Neurodevelopmental disorders: cluster 2 of the proposed meta-structure for DSM-V and ICD-11. Psychol Med, 39(12), 2013-2023.

Bailey, C. E., Manis, F. R., Pedersen, W. C., \& Seidenberg, M. S. (2004). Variation among developmental dyslexics: Evidence from a printed-word-learning task. Journal of experimental child psychology, 87(2), 125154.

Basse, I., Albaret, J.-M., \& Chaix, Y. (1999). Troubles psychomoteurs et dyslexie. Evolutions psychomotrices, 11(46), 207-213.

Beery, K. E. (1989). The developmental test of visual-motor integration (3rd ed.). Cleveland, OH: Modern Curriculum Press.

Bellocchi, S. (sous presse). Developmental Dyslexia, Visual Crowding and Eye Movements. In L. C. Stewart (Ed.), Eye Movement: Developmental Perspectives, Dysfunctions and Disorders in Humans. Hauppauge, NY: Nova Science Publishers, Inc.

Bellocchi, S., Muneaux, M., Bastien-Toniazzo, M., \& Ducrot, S. (2013). I can read it in your eyes: what eye movements tell us about visuo-attentional processes in developmental dyslexia. Res Dev Disabil, 34(1), 452460.

Bennett, I. J., Romano, J. C., Howard, J. H., Jr., \& Howard, D. V. (2008). Two forms of implicit learning in young adults with dyslexia. Annals of the New York Academy of Sciences, 1145, 184-198.

Berninger, V. W., Nielsen, K. H., Abbott, R. D., Wijsman, E., \& Raskind, W. (2008). Writing problems in developmental dyslexia: under-recognized and under-treated. Journal of school psychology, 46(1), 1-21.

Biotteau, M., Albaret, J.-M., Lelong, S., \& Chaix, Y. (2013). Cognitive Profile on the WISC-IV of Children With Developmental Coordination Disorder (DCD) and/or Developmental Dyslexia (DD). Brazilian Journal of Motor Behavior, 7(suppl), 104.

Biscaldi, M., Gezeck, S., \& Stuhr, V. (1998). Poor saccadic control correlates with dyslexia. Neuropsychologia, 36(11), 1189-1202.

Bishop, D. V., \& Snowling, M. J. (2004). Developmental dyslexia and specific language impairment: same or different? Psychol Bull, 130(6), 858-886.

Borella, E., Chicherio, C., Re, A. M., Sensini, V., \& Cornoldi, C. (2011). Increased intraindividual variability is a marker of ADHD but also of dyslexia: a study on handwriting. Brain and cognition, 77(1), 33-39.

Bosse, M. L., Tainturier, M. J., \& Valdois, S. (2007). Developmental dyslexia: the visual attention span deficit hypothesis. Cognition, 104(2), 198-230.

Brookes, R. L., Nicolson, R. I., \& Fawcett, A. J. (2007). Prisms throw light on developmental disorders. Neuropsychologia, 45(8), 1921-1930.

Bruininks, R. H. (1978). Bruininks-Oseretsky test of motor proficiency. Circle Pines, MN: American Guidance Service. 
Brun-Hénin, F. (2007). Trouble d'écriture chez le sujet dyslexique. Paper presented at the Journée Thématique Résodys "Coordination motrice et troubles des apprentissages".

Brun-Hénin, F., Velay, J. L., Beecham, Y., \& Cariou, S. (2013). Troubles d'écriture et dyslexie : revue théorique, aspects cliniques et apprroche expérimentale. Développements, 1, 4-28.

Bucci, M. P., Bremond-Gignac, D., \& Kapoula, Z. (2008). Poor binocular coordination of saccades in dyslexic children. Graefe's archive for clinical and experimental ophthalmology, 246(3), 417-428.

Chaix, Y., Albaret, J.-M., Brassard, C., Cheuret, E., de Castelnau, P., Benesteau, J., et al. (2007). Motor impairment in dyslexia: the influence of attention disorders. European journal of paediatric neurology, 11(6), 368-374.

Chaix, Y., Biotteau, M., Vayssiere, N., Lelong, S., \& Albaret, J.-M. (2013). An fMRI study of the procedural learning deficit hypothesis in Developmental Coordination Disorder and/or Developmental Dyslexia. Brazilian Journal of Motor Behavior, 7(suppl), 10.

Charles, M., Soppelsa, R., \& Albaret, J.-M. (2003). BHK - Echelle d'évaluation rapide de l'écriture chez l'enfant. Paris: Editions et Applications Psychologiques.

Chase, C., \& Stein, J. (2003). Visual magnocellular deficits in dyslexia. Brain, 126(Pt 9), E2; author reply E3.

Clements, S. D., \& Peters, J. E. (1962). Minimal Brain Dysfunctions in the School-Age Child: Diagnosis and Treatment. Archives of General Psychiatry, 6(3), 185-197.

Cornelissen, P., Richardson, A., Mason, A., Fowler, S., \& Stein, J. (1995). Contrast sensitivity and coherent motion detection measured at photopic luminance levels in dyslexics and controls. Vision Research, 35(10), $1483-1494$.

de Ajuriaguerra, J., Auzias, M., Coumes, I., Lavondes-Monod, V., Perron, R., \& Stamback, M. (1964). L'écriture chez l'enfant. Neuchâtel: Delachaux et Niestlé.

De Luca, M., Di Pace, E., Judica, A., Spinell, D., \& Zoccolotti, P. (1999). Eye movement patterns in linguistic and non-linguistic tasks in developmental surface dyslexia. Neuropsychologia, 37(12), 1407-1420.

Ducrot, S., \& Lété, B. (2008). Attention et contrôle oculaire en lecture experte. In G. Michael (Ed.), Neurosciences cognitives de l'attention visuelle (pp. 229-264). Marseille: Solal.

Ducrot, S., Lété, B., Bellocchi, S., Ghio, A., Pinton, F., \& Billard, C. (en préparation). Where dyslexics look first in a stimulus?

Ducrot, S., Lété, B., Sprenger-Charolles, L., Pynte, J., \& Billard, C. (2003). The Optimal Viewing Position Effect in Beginning and Dyslexic Readers. Current Psychology Letters, 1(10).

Ducrot, S., \& Pynte, J. (2002). What determines the eyes' landing position in words? Perception and Psychophysics, 64(7), 1130-1144.

Ducrot, S., Pynte, J., Ghio, A., \& Lete, B. (2013). Visual and linguistic determinants of the eyes' initial fixation position in reading development. Acta Psychol (Amst), 142(3), 287-298.

Eden, G. F., Stein, J. F., Wood, H. M., \& Wood, F. B. (1994). Differences in eye movements and reading problems in dyslexic and normal children. Vision Research, 34(10), 1345-1358.

Facoetti, A., Lorusso, M. L., Paganoni, P., Cattaneo, C., Galli, R., \& Mascetti, G. G. (2003). The time course of attentional focusing in dyslexic and normally reading children. Brain and cognition, 53(2), 181-184.

Facoetti, A., \& Molteni, M. (2001). The gradient of visual attention in developmental dyslexia. Neuropsychologia, 39(4), 352-357. 
Fawcett, A. J., \& Nicolson, R. I. (1992). Automatisation deficits in balance for dyslexic children. Percept Mot Skills, 75(2), 507-529.

Fawcett, A. J., \& Nicolson, R. I. (1995). Persistent deficits in motor skill of children with dyslexia. Journal of Motor Behavior, 27(3), 235-240.

Fawcett, A. J., \& Nicolson, R. I. (1999). Performance of Dyslexic Children on Cerebellar and Cognitive Tests. Journal of Motor Behavior, 31(1), 68-78.

Fawcett, A. J., Nicolson, R. I., \& Dean, P. (1996). Impaired performance of children with dyslexia on a range of cerebellar tasks. Annals of Dyslexia, 46, 259-283.

Fawcett, A. J., Nicolson, R. I., \& Maclagan, F. (2001). Cerebellar tests differentiate between groups of poor readers with and without IQ discrepancy. Journal of Learning Disabilities, 34(2), 119-135.

Fletcher-Flinn, C., Elmes, H., \& Strugnell, D. (1997). Visual-perceptual and phonological factors in the acquisition of literacy among children with congenital developmental coordination disorder. Developmental Medicine and Child Neurology, 39(3), 158-166.

Folia, V., Udden, J., Forkstam, C., Ingvar, M., Hagoort, P., \& Petersson, K. M. (2008). Implicit learning and dyslexia. Ann N Y Acad Sci, 1145, 132-150.

Gabay, Y., Schiff, R., \& Vakil, E. (2012a). Dissociation between online and offline learning in developmental dyslexia. J Clin Exp Neuropsychol, 34(3), 279-288.

Gabay, Y., Schiff, R., \& Vakil, E. (2012b). Dissociation between the procedural learning of letter names and motor sequences in developmental dyslexia. Neuropsychologia, 50(10), 2435-2441.

Geiger, G., Lettvin, J. Y., \& Zegarra-Moran, O. (1992). Task-determined strategies of visual process. Brain research. Cognitive brain research, 1(1), 39-52.

Geuze, R. H., \& Kalverboer, A. F. (1994). Tapping a Rhythm: A Problem of Timing for Children Who Are Clumsy and Dyslexic? Adapted Physical Activity Quarterly, 11(2), 203-213.

Gilger, J. W., \& Kaplan, B. J. (2001). Atypical brain development: A conceptual framework for understanding developmental learning disabilities. Developmental Neuropsychology, 20(2), 465-481.

Gilger, J. W., \& Wilkins, M. (2008). Atypical neurodevelopmental variation as a basis for learning disorders. In M. Mody \& E. Silliman (Eds.), Brain, behavior, and learning in language and reading disorders (pp. 7-40). New York: Guilford Press.

Gladstone, M., Best, C. T., \& Davidson, R. J. (1989). Anomalous bimanual coordination among dyslexic boys. Developmental Psychology, 25(2), 236-246.

Habib, M. (2000). The neurological basis of developmental dyslexia: an overview and working hypothesis. Brain, 123 Pt 12, 2373-2399.

Habib, M., \& Joly-Pottuz, B. (2008). Dyslexie, du diagnostic à la thérapeutique : un état des lieux. Revue de Neuropsychologie, 18(4), 247-325.

Haikio, T., Bertram, R., Hyona, J., \& Niemi, P. (2009). Development of the letter identity span in reading: evidence from the eye movement moving window paradigm. Journal of experimental child psychology, 102(2), 167-181.

Haslum, M. N., \& Miles, T. R. (2007). Motor performance and dyslexia in a national cohort of 10-year-old children. Dyslexia: An International Journal of Research and Practice, 13(4), 257-275.

Hawelka, S., Gagl, B., \& Wimmer, H. (2010). A dual-route perspective on eye movements of dyslexic readers. Cognition, 115(3), 367-379. 
Henderson, J. M., \& Ferreira, F. (1990). Effects of foveal processing difficulty on the perceptual span in reading: implications for attention and eye movement control. Journal of experimental psychology. Learning, memory, and cognition, 16(3), 417-429.

Henderson, S. E., \& Hall, D. (1982). Concomitants of clumsiness in young schoolchildren. Developmental Medicine and Child Neurology, 24(4), 448-460.

Henderson, S. E., \& Sugden, D. A. (1992). Movement Assessment Battery for Children. Londres: The Psychological Corporation.

Ho, C. S.-H., Chan, D. W.-O., Leung, P. W. L., Lee, S.-H., \& Tsang, S.-M. (2005). Reading-related cognitive deficits in developmental dyslexia, attention-deficit/hyperactivity disorder, and developmental coordination disorder among Chinese children. Reading Research Quarterly, 40(3), 318-337.

Huau, A., Jover, M., \& Velay, J. L. (en préparation). Is learning to write difficult for children with developmental dyslexia?

Huau, A., Velay, J.-L., \& Jover, M. (2012). Etude de la graphomotricité chez des enfants présentant une dyslexie. Paper presented at the Les troubles du langage écrit de l'enfance à l'âge adulte.

Hutzler, F., Kronbichler, M., Jacobs, A. M., \& Wimmer, H. (2006). Perhaps correlational but not causal: no effect of dyslexic readers' magnocellular system on their eye movements during reading. Neuropsychologia, 44(4), 637-648.

Hutzler, F., \& Wimmer, H. (2004). Eye movements of dyslexic children when reading in a regular orthography. Brain and Language, 89(1), 235-242.

INSERM. (2007). Dyslexie, dysorthographie, dyscalculie : bilan des données scientifiques. Paris: INSERM.

Iversen, S., Berg, K., Ellertsen, B., \& Tonnessen, F. E. (2005). Motor coordination difficulties in a municipality group and in a clinical sample of poor readers. Dyslexia, 11(3), 217-231.

Jover, M. (2012). Trouble de l'acquisition de la coordination et troubles de l'écriture : peut-on parler de comorbidité ? Développements, 12, 19-25.

Jover, M., Huau, A., \& Velay, J.-L. (2011). Graphomotor abilities in developmental coordination disorder and reading disability. Paper presented at the IXth Developmental Coordination Disorder International Conference.

Kaplan, B. J., Crawford, S., Cantell, M., Kooistra, L., \& Dewey, D. (2006). Comorbidity, co-occurrence, continuum: What's in a name? Child Care and Health Development, 32(6), 723-731.

Kaplan, B. J., Dewey, D. M., Crawford, S. G., \& Wilson, B. N. (2001). The term comorbidity is of questionable value in reference to developmental disorders: Data and theory. Journal of Learning Disabilities, 34(6), 555565.

Kaplan, B. J., Wilson, B. N., Dewey, D., \& Crawford, S. G. (1998). DCD may not be a discrete disorder. Human Movement Science, 17(4-5), 471-490.

Kapoula, Z., Bucci, M. P., Jurion, F., Ayoun, J., Afkhami, F., \& Bremond-Gignac, D. (2007). Evidence for frequent divergence impairment in French dyslexic children: deficit of convergence relaxation or of divergence per se? Graefe's archive for clinical and experimental ophthalmology, 245(7), 931-936.

Kavale, K., \& Mattson, P. D. (1983). "One jumped off the balance beam": meta-analysis of perceptual-motor training. Journal of learning disabilities, 16(3), 165-173.

Kirkby, J. A., Blythe, H. I., Drieghe, D., \& Liversedge, S. P. (2011). Reading text increases binocular disparity in dyslexic children. PLOS One, 6(11), e27105. 
Kooistra, L., Crawford, S., Dewey, D., Cantell, M., \& Kaplan, B. J. (2005). Motor correlates of ADHD: Contribution of reading disability and oppositional defiant disorder. Journal of Learning Disabilities, 38(3), 195206.

Lam, S. S., Au, R. K., Leung, H. W., \& Li-Tsang, C. W. (2011). Chinese handwriting performance of primary school children with dyslexia. Res Dev Disabil, 32(5), 1745-1756.

Launay, L., \& Valdois, S. (2004). Evaluation et prise en charge cognitive de l'enfant dyslexique et/ou dysorthographique de surface. In S. Valdois, P. Colé \& D. David (Eds.), Apprentissage de la lecture et dyslexie développementale, de la théorie à la pratique (pp. 209-232). Marseille: Solal.

Lefavrais, P. (2005). Alouette-R Test d'analyse de la lecture et de la dyslexie. Paris: ECPA.

Lehtimaki, T. M., \& Reilly, R. G. (2005). Improving eye movement control in young readers. Artificial Intelligence Review, 24(3-4), 477-488.

Leslie, S. C., Davidson, R. J., \& Batey, O. B. (1985). Purdue pegboard performance of disabled and normal readers: unimanual versus bimanual differences. Brain and Language, 24(2), 359-369.

Lobier, M., \& Valdois, S. (2010). Prise en charge des dyslexies développementales : critères d'évaluation. Revue de neuropsychologie, 1(2), 102-109.

Lovegrove, W. J., Martin, F., \& Slaghuis, W. L. (1986). A theoretical and experimental case of a visual deficit in specific reading disability. Cognitive neuropsychology, 3(2), 225-267.

Mæland, A. F., \& Søvik, N. (1993). Children with motor coordination problems and learning disabilities in reading, spelling, writing and arithmetic. European Journal of Special Needs Education, 8(2), 81-98.

Martelli, M., Di Filippo, G., Spinelli, D., \& Zoccolotti, P. (2009). Crowding, reading, and developmental dyslexia. Journal of Vision, 9(4), 14 11-18.

McPhillips, M., \& Sheehy, N. (2004). Prevalence of persistent primary reflexes and motor problems in children with reading difficulties. Dyslexia, 10(4), 316-338.

Menghini, D., Hagberg, G. E., Petrosini, L., Bozzali, M., Macaluso, E., Caltagirone, C., et al. (2008). Structural correlates of implicit learning deficits in subjects with developmental dyslexia. Ann N Y Acad Sci, 1145, 212221.

Moe-Nilssen, R., Helbostad, J. L., Talcott, J. B., \& Toennessen, F. E. (2003). Balance and gait in children with dyslexia. Experimental and Brain Research, 150(2), 237-244.

Moore, L. H., Brown, W. S., Markee, T. E., Theberge, D. C., \& Zvi, J. C. (1995). Bimanual coordination in dyslexic adults. Neuropsychologia, 33(6), 781-793.

Needle, J. L., Fawcett, A. J., \& Nicolson, R. I. (2006). Balance and dyslexia: An investigation of adults' abilities. European Journal of Cognitive Psychology, 18(6), 909-936.

Newell, A. (1973). You can't play 20 questions with nature and win: commebnts on the symposium. In W. Chase (Ed.), Visual information processing (pp. 283-308). London: Academic Press.

Nicolson, R. I., \& Fawcett, A. J. (1990). Automaticity: a new framework for dyslexia research? Cognition, 35(2), 159-182.

Nicolson, R. I., \& Fawcett, A. J. (1994). Comparison of deficits in cognitive and motor skills among children with dyslexia. Annals of Dyslexia, 44, 147-164.

Nicolson, R. I., \& Fawcett, A. J. (1995). Dyslexia is more than a phonological disability. Dyslexia, 1, 19-36. 
Nicolson, R. I., \& Fawcett, A. J. (1999). Developmental dyslexia: The role of the cerebellum1. Dyslexia, 5(3), 155-177.

Nicolson, R. I., \& Fawcett, A. J. (2005). Developmental dyslexia, learning and the cerebellum. Journal of neural transmission. Supplementum(69), 19-36.

Nicolson, R. I., \& Fawcett, A. J. (2006). Do cerebellar deficits underlie phonological problems in dyslexia? Developmental Science, 9(3), 259-262; discussion 265-259.

Nicolson, R. I., \& Fawcett, A. J. (2007). Procedural learning difficulties: reuniting the developmental disorders? Trends in Neurosciences, 30(4), 135-141.

Nicolson, R. I., \& Fawcett, A. J. (2011). Dyslexia, dysgraphia, procedural learning and the cerebellum. Cortex, 47(1), 117-127.

Nicolson, R. I., Fawcett, A. J., Brookes, R. L., \& Needle, J. (2010). Procedural learning and dyslexia. Dyslexia, 16(3), 194-212.

Nicolson, R. I., Fawcett, A. J., \& Dean, P. (2001). Developmental dyslexia: the cerebellar deficit hypothesis. Trends in Neurosciences, 24(9), 508-511.

O'Hare, A., \& Khalid, S. (2002). The association of abnormal cerebellar function in children with developmental coordination disorder and reading difficulties. Dyslexia, 8(4), 234-248.

O'Regan, J. K., Levy-Schoen, A., Pynte, J., \& Brugaillere, B. (1984). Convenient fixation location within isolated words of different length and structure. Journal of experimental psychology. Human perception and performance, 10(2), 250-257.

Organisation Mondiale de la Santé, O. M. S. (1994). CIM-10, Classification internationale des troubles mentaux et des troubles du comportement : descriptions cliniques et directives pour le diagnostic. Paris: Masson.

Paz-Villagrán, V., Gilhodes, J. C., \& Velay, J. L. (2013). Les enfants dysgraphiques sont-ils réellement plus lents que les autres? Développements, 1, 38-44.

Pennington, B. F. (2006). From single to multiple deficit models of developmental disorders. Cognition, 101(2), 385-413.

Pennington, B. F., Santerre-Lemmon, L., Rosenberg, J., MacDonald, B., Boada, R., Friend, A., et al. (2012). Individual prediction of dyslexia by single versus multiple deficit models. J Abnorm Psychol, 121(1), 212-224.

Peterson, R. L., Pennington, B. F., \& Olson, R. K. (2013). Subtypes of developmental dyslexia: Testing the predictions of the dual-route and connectionist frameworks. Cognition, 126(1), 20-38.

Poblano, A., Ishiwara, K., de Lourdes Arias, M., Garcia-Pedroza, F., Marin, H., \& Trujillo, M. (2002). Motor control alteration in posturography in learning-disabled children. Archives of medical research, 33(5), 485-488.

Polatajko, H., Fox, M., \& Missiuna, C. (1995). An International Consensus on Children with Developmental Coordination Disorder. Canadian Journal of Occupational Therapy, 62(1), 3-6.

Pozzo, T., Vernet, P., Creuzot-Garcher, C., Robichon, F., Bron, A., \& Quercia, P. (2006). Static postural control in children with developmental dyslexia. Neuroscience Letters, 403(3), 211-215.

Prado, C., Dubois, M., \& Valdois, S. (2007). The eye movements of dyslexic children during reading and visual search: impact of the visual attention span. Vision Research, 47(19), 2521-2530.

Raberger, T., \& Wimmer, H. (2003). On the automaticity/cerebellar deficit hypothesis of dyslexia: balancing and continuous rapid naming in dyslexic and ADHD children. Neuropsychologia, 41(11), 1493-1497. 
Ramus, F., Pidgeon, E., \& Frith, U. (2003). The relationship between motor control and phonology in dyslexic children. Journal of Child Psychology and Psychiatry, 44(5), 712-722.

Ramus, F., Rosen, S., Dakin, S. C., Day, B. L., Castellote, J. M., White, S., et al. (2003). Theories of developmental dyslexia: insights from a multiple case study of dyslexic adults. Brain, 126(Pt 4), 841-865.

Rayner, K. (1979). Eye guidance in reading: fixation locations within words. Perception, 8(1), 21-30.

Rayner, K. (1986). Eye movements and the perceptual span in beginning and skilled readers. Journal of experimental child psychology, 41(2), 211-236.

Rayner, K. (1998). Eye movements in reading and information processing: 20 years of research. Psychological Bulletin, 124(3), 372-422.

Rayner, K., Pollatsek, A., \& Bilsky, A. B. (1995). Can a temporal processing deficit account for dyslexia ? Psychonomic bulletin \& review, 3, 238-244.

Rayner, K., Slattery, T. J., \& Belanger, N. N. (2010). Eye movements, the perceptual span, and reading speed. Psychonomic bulletin \& review, 17(6), 834-839.

Rochelle, K. S., \& Talcott, J. B. (2006). Impaired balance in developmental dyslexia? A meta-analysis of the contending evidence. Journal of child psychology and psychiatry, and allied disciplines, 47(11), 1159-1166.

Soppelsa, R., \& Albaret, J.-M. (2004). Manuel de la Batterie d'Evaluation du Mouvement chez l'Enfant $(M A B C)$. Paris: Editions du Centre de Psychologie Appliquée.

Stein, J. (1988). Dyslexia. BMJ, 297(6652), 854.

Stein, J. (2001). The magnocellular theory of developmental dyslexia. Dyslexia, 7(1), 12-36.

Stein, J., \& Walsh, V. (1997). To see but not to read; the magnocellular theory of dyslexia. Trends in Neurosciences, 20(4), 147-152.

Stoodley, C. J., Fawcett, A. J., Nicolson, R. I., \& Stein, J. F. (2005). Impaired balancing ability in dyslexic children. Experimental Brain Research, 167(3), 370-380.

Stoodley, C. J., Fawcett, A. J., Nicolson, R. I., \& Stein, J. F. (2006). Balancing and pointing tasks in dyslexic and control adults. Dyslexia, 12(4), 276-288.

Stoodley, C. J., Harrison, E. P., \& Stein, J. F. (2006). Implicit motor learning deficits in dyslexic adults. Neuropsychologia, 44(5), 795-798.

Stoodley, C. J., \& Stein, J. F. (2006). A processing speed deficit in dyslexic adults? Evidence from a pegmoving task. Neuroscience Letters, 399(3), 264-267.

Stoodley, C. J., \& Stein, J. F. (2011). The cerebellum and dyslexia. Cortex, 47(1), 101-116.

Stoodley, C. J., \& Stein, J. F. (2013). Cerebellar function in developmental dyslexia. Cerebellum, 12(2), 267276.

Valdois, S., Lassus-Sangosse, D., \& Lobier, M. (2012). The visual nature of the visual attention span disorder in developmental dyslexia. In J. Stein \& Z. Kapoula (Eds.), Visual aspects of dyslexia (pp. 111-122). Oxford: Oxford University Press.

van Galen, G. P. (1991). Handwriting: Issues for a psychomotor theory. Human Movement Science, 10(2-3), 165-191.

Velay, J.-L., Daffaure, V., Giraud, K., \& Habib, M. (2002). Interhemispheric sensorimotor integration in pointing movements: a study on dyslexic adults. Neuropsychologia, 40(7), 827-834. 
Velay, J.-L., Henin, F., Moulin, C., Thomas, T., Devos-Charles, I., \& Habib, M. (2009). Handwriting Variability in children writing letters: A study in dyslexics, dysgraphics and proficient handwriters. Paper presented at the 14th Conference of the International Graphonomics Society.

Vicari, S., Finzi, A., Menghini, D., Marotta, L., Baldi, S., \& Petrosini, L. (2005). Do children with developmental dyslexia have an implicit learning deficit? J Neurol Neurosurg Psychiatry, 76(10), 1392-1397.

Vicari, S., Marotta, L., Menghini, D., Molinari, M., \& Petrosini, L. (2003). Implicit learning deficit in children with developmental dyslexia. Neuropsychologia, 41(1), 108-114.

Vieira, S., Quercia, P., Michel, C., Pozzo, T., \& Bonnetblanc, F. (2009). Cognitive demands impair postural control in developmental dyslexia: a negative effect that can be compensated. Neuroscience Letters, 462(2), 125-129.

Viholainen, H., Ahonen, T., Lyytinen, P., Cantell, M., Tolvanen, A., \& Lyytinen, H. (2006). Early motor development and later language and reading skills in children at risk of familial dyslexia. Developmental Medicine and Child Neurology, 48(5), 367-373.

Visser, J. (2003). Developmental coordination disorder: a review of research on subtypes and comorbidities. Human Movement Science, 22(4-5), 479-493.

Vuijk, P. J., Hartman, E., Mombarg, R., Scherder, E., \& Visscher, C. (2011). Associations between academic and motor performance in a heterogeneous sample of children with learning disabilities. Journal of Learning Disabilities, 44(3), 276-282.

Wimmer, H., Mayringer, H., \& Lander, K. (1998). Poor reading : a deficit in skill-automatization or a phonological deficit? Scientific Studies of Reading, 2(4), 321-340.

Wimmer, H., Mayringer, H., \& Raberger, T. (1999). Reading and dual-task balancing: Evidence against the automatization deficit explanation of developmental dyslexia. Journal of Learning Disabilities, 32(5), 473-478.

Yap, R. L., \& van der Leij, A. (1994). Testing the automatization deficit hypothesis of dyslexia via a dual-task paradigm. Journal of Learning Disabilities, 27(10), 660-665.

Zeffiro, T., \& Eden, G. (2001). The cerebellum and dyslexia: perpetrator or innocent bystander? Trends in Neurosciences, 24(9), 512-513.

Zorzi, M., Barbiero, C., Facoetti, A., Lonciari, I., Carrozzi, M., Montico, M., et al. (2012). Extra-large letter spacing improves reading in dyslexia. Proceedings of the National Academy of Sciences of the United States of America, 109(28), 11455-11459. 